\title{
Uso dos serviços de saúde e adesão ao distanciamento social por adultos com doenças crônicas na pandemia de COVID-19, Brasil, 2020
}

\author{
Use of health services and adherence to social distancing by adults \\ with Noncommunicable Diseases during the COVID-19 pandemic, \\ Brazil, 2020
}

Deborah Carvalho Malta (https://orcid.org/0000-0002-8214-5734) ${ }^{1}$

Crizian Saar Gomes (https://orcid.org/0000-0001-6586-4561) ${ }^{2}$

Alanna Gomes da Silva (https://orcid.org/0000-0003-2587-5658) ${ }^{2}$

Laís Santos de Magalhães Cardoso (http://orcid.org/0000-0002-1114-5470) ${ }^{2}$

Marilisa Berti de Azevedo Barros (https://orcid.org/0000-0003-3974-195X) ${ }^{3}$

Margareth Guimarães Lima (https://orcid.org/0000-0001-6996-0745) ${ }^{3}$

Paulo Roberto Borges de Souza Junior (https://orcid.org/0000-0002-8142-4790) ${ }^{4}$

Célia Landmann Szwarcwald (https://orcid.org/0000-0002-7798-2095) ${ }^{4}$

${ }^{1}$ Departamento de Enfermagem MaternoInfantil e Saúde Pública, Universidade Federal de Minas Gerais. Av. Alfredo Balena $1905^{\circ}$ andar, Escola de Enfermagem, Santa Efigênia. 30130100 Belo Horizonte MG Brasil. dcmalta@uol.com.br ${ }^{2}$ Programa de PósGraduação em Enfermagem, Escola de Enfermagem, Universidade Federal de Minas Gerais. Belo Horizonte MG Brasil. ${ }^{3}$ Departamento de Saúde Coletiva, Faculdade de Ciências Médicas, Universidade Estadual de Campinas. Campinas São Paulo Brasil.

${ }^{4}$ Instituto de Comunicação

e Informação Científica e Tecnológica em Saúde, Fundação Oswaldo Cruz. Rio de Janeiro RJ Brasil.

\begin{abstract}
The present study investigates the association between the self-reported diagnosis of noncommunicable disease (NCD) and the adherence to social distancing and the use of health services during the COVID-19 pandemic. This was a cross-sectional study with Brazilian adults who participated in the ConVid-Behavior Survey, conducted online between April 24 and May 24, 2020( $n=$ 45.161). This studyconsidered the following NCDs. diabetes, hypertension, respiratory disease, heart disease, and cancer, and evaluated the use of heal th services and the adherence to social distancing, as well as estimated the prevalences and adjusted prevalence ratio (aPR); 33,9\% (95\% CI: 32,5$35,3)$ referred to one or more NCD. Individuals with NCDsshowed a greater adherence to intense social distancing (aPR: 1,07;95\% CI: 1,03-1,11), sought out health services more often (aPR:1,24; 95\% CI:1,11-1,38), and found greater difficultyin scheduling doctor's appointments (aPR:1.52; 95\% CI 1,35-1,71), receiving healthcare treatment (APR:1,50;95\% CI:1,22-1,84) and medication (APR:2,17;95\% CI:1,77-2,67), and performing examinations (APR:1,78;95\% CI:1,50-2,10) and scheduled interventions (APR:1,65;95\% CI:1,16 2,34). The presence of NCDs was associated with social distancing, seeking out health care, and difficulty in using health services.
\end{abstract}

Key words Quarantine, Infection by coronavirus, Noncommunicable diseases, Access to health services
Resumo Este estudo investiga a associação entre diagnóstico autorreferido de Doença Crônica Não Transmissivel (DCNT) e adesão ao distanciamento social e utilização dos serviços de saúde durante a pandemia de COVID-19. Estudo transversal com adultos brasileiros que participaram da ConVid Pesquisa de Comportamentos, realizada de 24 de abril a 24 de maio de 2020, via web ( $n=$ 45.161). Considerou as DCNT: diabetes, hipertensão, doença respiratória, doença do coração e câncer. Avaliou a utilização de serviços de saúde e a adesão ao distanciamento social. Estimou as prevalências e razões de prevalências ajustadas ( $\mathrm{RPa}$ ). 33,9\% (IC95\%: 32,5-35,3) referiu uma ou mais DCNT. Indivíduos com DCNT tiveram maior adesão ao distanciamento social intenso (RPa:1,07; IC95\%:1,03-1,11), procuraram mais o serviço de saúde (RPa:1,24; IC95\%:1,11-1,38) e tiveram mais dificuldades para marcar consulta (RPa:1,52; IC95\%:1,35-1,71), conseguir atendimento de saúde (RPa:1,50; IC95\%:1,22-1,84) e medicamentos (RPa:2,17; IC95\%:1,77-2,67), realizar exames ( $R P a: 1,78$; IC95\%:1,50-2,10) $e$ intervenções programadas (RPa:1,65; IC95\%:1,162,34). A presença de DCNT associou-se à maior adesão ao distanciamento social, procura por atendimento de saúde e dificuldade na utilização dos serviços de saúde.

Palavras-chave Quarentena; Infecções por coronavírus; Doenças não transmissíveis; Acesso aos serviços de saúde 


\section{Introdução}

As Doenças Crônicas Não Transmissíveis (DCNT) são as principais causas de morte em todo o mundo, responsáveis por $71 \%$ (41 milhões) dos óbitos globais em 2016, e configuram um problema de saúde pública, devido ao aumento do custo econômico e social, da morbidade, do tempo de internação e da mortalidade ${ }^{1}$.

$\mathrm{Na}$ pandemia de COVID-19 (Coronavírus Disease 2019), as DCNT se tornam ainda mais preocupantes, visto que essas doenças e seus fatores de risco comportamentais e metabólicos agravam os casos, aumentam o tempo de internação e as taxas de mortalidade pela COVID- $19^{2}$. Além disso, indivíduos com DCNT são mais acometidos pelas formas severas da COVID-19, que requerem hospitalização, cuidados intensivos e uso de ventiladores mecânicos ${ }^{2}$. Estudo na Itália revelou que 96,2\% das pessoas que morreram devido à COVID-19 apresentavam hipertensão $(69,2 \%)$, diabetes tipo $2(31,8 \%)$, doença cardíaca isquêmica $(28,2 \%)$, doença pulmonar obstrutiva crônica $(16,9 \%)$ e câncer $(16,3 \%)^{3}$.

A adoção do distanciamento social, recomendado pela Organização Mundial da Saúde (OMS) como uma das medidas não farmacológicas que contribuem para diminuir a propagação e a transmissão da COVID-19 ${ }^{4}$, pode, por outro lado, acarretar efeitos negativos à saúde, principalmente nas pessoas com DCNT. O distanciamento social pode resultar em mudanças nas rotinas diárias, no estilo de vida, como redução de atividade física, aumento do consumo do tabaco e bebidas alcóolicas ${ }^{5}$, além de comprometer o acesso aos serviços de saúde ${ }^{2,6-7}$ e, consequentemente, a continuidade do cuidado prestado a indivíduos com DCNT, e propiciar o agravamento do seu estado de saúde.

Estudo realizado pela Organização Pan-Americana da Saúde (OPAS) revelou que os serviços de prevenção e tratamento de DCNT foram afetados pela pandemia de COVID-19 na região das Américas ${ }^{8}$. Houve reorganização ou descontinuação de serviços de saúde de rotina, com interrupção da assistência a pessoas em tratamento de doenças como câncer, doenças cardiovasculares e diabetes $^{8}$. Adicionalmente, profissionais de saúde atuantes na atenção à saúde de indivíduos com DCNT foram redirecionados para o trabalho em saúde no campo das ações de contingenciamento da COVID-198.

Nesse contexto, a redução ou mesmo a interrupção dos serviços de saúde de rotina constitui ameaça à saúde das pessoas que vivem com
DCNT e pode acarretar "uma epidemia paralela de mortes evitáveis"8. Por isso, torna-se necessário investigar o uso de serviços durante a pandemia de COVID-19 por pessoas com DCNT.

Face ao exposto, o objetivo deste estudo consistiu em investigar se o diagnóstico autorreferido de DCNT está associado à adesão ao distanciamento social e à utilização dos serviços de saúde durante a pandemia de COVID-19 no Brasil.

\section{Métodos}

\section{Desenho do estudo e processo amostral}

Trata-se de um estudo transversal realizado com indivíduos adultos, com idade igual ou maior que 18 anos, residentes no Brasil, que participaram do inquérito de saúde virtual "ConVid - Pesquisa de Comportamentos”. A ConVid foi conduzida pela Fundação Oswaldo Cruz (Fiocruz) em parceria com a Universidade Federal de Minas Gerais (UFMG) e a Universidade Estadual de Campinas (Unicamp), com objetivo de avaliar mudanças nos estilos de vida e nos cuidados à saúde dos brasileiros durante a pandemia de COVID-19.

A coleta de dados ocorreu de 24 de abril a 24 de maio de 2020, a partir do preenchimento de um questionário online. Todas as pessoas com 18 anos ou mais residentes no território brasileiro durante a Pandemia de COVID-19 foram elegíveis para participar deste estudo.

Os participantes foram convidados a participar do estudo por meio de um procedimento de amostragem em cadeia ${ }^{10}$. Primeiramente, os pesquisadores responsáveis pelo estudo selecionaram um grupo de pesquisadores (chamados de influenciadores) de diferentes estados brasileiros para desencadear a rede de convidados; após responderem ao questionário, os influenciadores enviaram o link da pesquisa para pelo menos 20 pessoas das suas redes sociais, obedecendo a uma estratificação por sexo, faixa de idade ( 18 a 39; 40 a $59 ; 60$ ou mais) e grau de escolaridade (Ensino Médio incompleto ou menos; Ensino Médio completo ou mais). A cada uma dessas pessoas que recebeu o link foi solicitado que convidasse pelo menos outras três pessoas de suas redes sociais, por meio da mensagem ao final do questionário: "Faça parte da Rede ConVid e compartilhe esta pesquisa com três ou mais convidados da sua rede social. Você pode fazer isso clicando aqui ou copiando e enviando o nosso link https://convid. fiocruz.br". 
As informações foram coletadas diretamente pela internet e armazenadas no servidor do Instituto de Comunicação e Informação Científica e Tecnológica em Saúde da Fiocruz, sem possibilidade de identificação dos participantes.

Mais detalhes sobre a pesquisa podem ser encontrados no site oficial da Convid - Pesquisa de Comportamentos?.

\section{Variáveis do estudo}

O diagnóstico autorreferido de DCNT foi definido pela resposta positiva para presença de uma ou mais DCNT dentre as seguintes: diabetes; hipertensão; doença respiratória; doença do coração; câncer. A pergunta no questionário web se referia ao diagnóstico médico prévio dessas doenças: "Algum médico já lhe deu o diagnóstico de alguma dessas doenças?”.

Para avaliar o distanciamento social intenso foi utilizada a pergunta: "Durante a pandemia do novo coronavírus, com que intensidade você fez (ou ainda está fazendo) restrição do contato com as pessoas?". As opções de respostam eram: a) não fiz nada, levei vida normal; b) procurei tomar cuidados, ficar à distância das pessoas, reduzir um pouco o contato, não visitar idosos, mas continuei trabalhando e saindo; c) fiquei em casa só saindo para compras em supermercado e farmácia; d) fiquei rigorosamente em casa, saindo só por necessidades de atendimento à saúde. A variável foi categorizada em "não" (opções "a e b") e sim (opções "c e d").

A utilização de serviços de saúde foi avaliada segundo procura pelo serviço de saúde e dificuldades no acesso aos serviços. Para avaliar a procura dos serviços foi considerada a seguinte pergunta: "Durante a pandemia de coronavírus, procurou atendimento de saúde com um médico, dentista ou outro profissional de saúde?". A resposta era "sim" ou "não". Com relação às dificuldades no acesso aos serviços, considerou-se a questão: "No período da pandemia, você teve alguma dessas dificuldades relacionadas aos cuidados à sua saúde?": a) marcar consulta; b) conseguir atendimento de saúde; c) conseguir medicamentos; d) realizar exames solicitados; e) realizar intervenções programadas; f) conseguir vaga para internação. As opções de resposta eram "sim" ou "não".

As seguintes variáveis sociodemográficas foram analisadas: sexo (masculino e feminino), faixa etária (18 a 39; 40 a 59 e 60 anos ou mais) e escolaridade (Ensino Médio incompleto; Ensino Médio completo; e Ensino Superior completo).

\section{Análise dos dados}

A amostragem por redes não é probabilística, não sendo possível, portanto, calcular os pesos naturais do desenho de amostragem. Por esse motivo, de modo a possibilitar a obtenção de uma amostra representativa e a mesma distribuição da população brasileira identificada na Pesquisa Nacional de Amostra por Domicílios (PNAD) em 2019, do Instituto Brasileiro de Geografia e Estatística (IBGE), foram utilizados procedimentos de pós-estratificação ${ }^{11}$ por: Unidade da Federação (UF), capital, sexo, faixa de idade (18 a 29; 30 a 39; 40 a 49; 50 a 59; 60 anos ou mais), grau de escolaridade (Ensino Superior incompleto; Ensino Superior completo) e raça/ cor da pele ${ }^{12}$. Essa técnica corrige a sub ou sobre representação de segmentos populacionais e tem sido utilizada frequentemente em pesquisas domiciliares, como na Pesquisa Nacional por Amostra de Domicílios Contínua (PNAD-Contínua) e no Inquérito Telefônico de Vigilância de Fatores de Risco e Proteção para Doenças Crônicas (Vigitel) $)^{13}$.

Realizou-se análise descritiva das variáveis por meio do cálculo de frequências relativas e intervalos de confiança de 95\% (IC95\%). Para avaliar a associação entre diagnóstico autorreferido de uma ou mais DCNT, utilização dos serviços de saúde e adesão às medidas de restrição social, foram calculadas as razões de prevalência (RP) brutas e ajustadas por sexo, faixa etária e escolaridade, bem como seus respectivos IC95\%, utilizando-se modelos de regressão de Poisson com variância robusta.

O processamento das informações foi realizado no Stata versão 14, empregando o módulo survey, que considera os pesos de pós-estratificação.

\section{Aspectos éticos}

Esta pesquisa fundamentou-se na Resolução 466/2012 do Conselho Nacional de Saúde e foi aprovada pela Comissão Nacional de Ética em Pesquisa. Os participantes preencheram o Termo de Consentimento Livre e Esclarecido, todas as respostas foram anônimas e sem qualquer tipo de identificação.

\section{Resultados}

Participaram do estudo 47.184 pessoas e foram excluídos $2.023(4,3 \%)$ questionários, cujas in- 
formações necessárias para a calibração dos dados não haviam sido preenchidas, ou seja, questionários sem informação sobre unidade federativa, sexo, idade, raça/cor da pele ou escolaridade, totalizando uma amostra de 45.161 entrevistados em todo o Brasil.

A maioria dos participantes era do sexo feminino (53,6\%; IC95\%: 52,0 - 55,0), tinha entre 18 e 39 anos (45,7\%; IC95\%: 44,2-47,1), Ensino Médio completo (72,4\%; IC95\%: 71,3-73,5) e cor da pele parda (45,7\%; IC95\%: 44,2-47,2) e branca $(45,2 \% ; 43,8-46,6)$; (Tabela 1 ).

A prevalência de uma ou mais DCNT foi de 33,9\% (IC95\%: 32,5-35,3), sendo maior entre os idosos (58,6\%; IC95\%: 55,3-61,9), e nos indivíduos com Ensino Médio incompleto (39,3\%; IC95\%: 34,4-44,5). O diagnóstico prévio autorreferido de hipertensão, diabetes, doença respiratória, doença do coração e câncer foi reportado por $19,3 \%, 7,2 \%, 11,3 \%, 4,3 \%$ e $2,4 \%$ dos participantes, respectivamente, sendo maior entre os idosos, exceto para doenças respiratórias, cuja prevalência foi maior nos mais jovens (18 a 39 anos). Indivíduos com maior escolaridade (superior completo) apresentaram menor prevalência de diagnóstico autorreferido de hipertensão, diabetes e doença do coração. As prevalências foram semelhantes entre sexos masculino e feminino (Tabela 2).
Ao analisar a adesão ao distanciamento social intenso, verificou-se que os indivíduos com uma ou mais DCNT foram os que referiram maior adesão (RPa: 1,07; IC95\%: 1,04 - 1,11) em comparação com aqueles sem DCNT (Figura 1).

Tabela 1. Características sociodemográficas dos participantes do estudo. Brasil. ConVid - Pesquisa de Comportamentos, 2020.

\begin{tabular}{lr}
\hline \multicolumn{1}{c}{ Variáveis } & \multicolumn{1}{c}{$\%(\mathbf{I C 9 5 \% )}$} \\
\hline Sexo & \\
Masculino & $46,4(45,0-47,9)$ \\
Feminino & $53,6(52,1-55,0)$ \\
Faixa etária & \\
18 a 39 anos & $45,7(44,2-47,1)$ \\
40 a 59 anos & $34,0(32,7-35,4)$ \\
60 anos ou mais & $20,3(19,1-21,6)$ \\
Escolaridade & \\
Ensino Médio incompleto & $11,1(10,1-12,3)$ \\
Ensino Médio completo & $72,4(71,3-73,5)$ \\
Ensino Superior completo & $16,5(16,0-17,0)$ \\
Raça/cor & \\
Branca & $45,2(43,8-46,6)$ \\
Preta & $8,3(7,5-9,2)$ \\
Parda & $45,7(44,2-47,2)$ \\
Outra & $0,8(0,6-0,9)$ \\
\hline Nota: IC95\% = Intervalo de confianca de 95\%.
\end{tabular}

Nota: IC95\% = Intervalo de confiança de 95\%.

Fonte: ConVid - Pesquisa de Comportamentos, 2020.

Tabela 2. Prevalência de diagnóstico autorreferido de uma ou mais DCNT, hipertensão, diabetes, doença respiratória, doença do coração ou câncer, segundo sexo, faixa etária e escolaridade. Brasil. ConVid - Pesquisa de Comportamentos, 2020 .

\begin{tabular}{|c|c|c|c|c|c|c|}
\hline \multirow[t]{2}{*}{ Variáveis } & $\begin{array}{l}\text { Ter uma ou } \\
\text { mais DCNT }\end{array}$ & Hipertensão & Diabetes & $\begin{array}{c}\text { Doença } \\
\text { respiratória }\end{array}$ & $\begin{array}{l}\text { Doença do } \\
\text { Coração }\end{array}$ & Câncer \\
\hline & \% (IC95\%) & $\%($ IC95\%) & $\%$ (IC95) & \% (IC95\%) & $\%($ IC95\%) & $\%($ IC95\%) \\
\hline Total & $33,9(32,5-35,3)$ & $19,3(18,2-20,4)$ & $7,2(6,5-8,0)$ & $11,3(10,4-12,2)$ & $4,3(3,7-5,0)$ & $2,4(2,1-2,9)$ \\
\hline \multicolumn{7}{|l|}{ Sexo } \\
\hline Masculino & $33,3(31,0-35,7)$ & $19,7(17,9-21,7)$ & $7,7(6,5-9,1)$ & $10,2(8,8-11,8)$ & $4,8(3,8-6,1)$ & $2,0(1,4-2,8)$ \\
\hline Feminino & $34,4(32,8-35,9)$ & $18,9(17,6-20,2)$ & $6,8(5,9-7,8)$ & $12,3(11,3-13,3)$ & $3,9(3,2-4,8)$ & $2,8(2,4-3,3)$ \\
\hline \multicolumn{7}{|l|}{ Faixa etária } \\
\hline 18 a 39 anos & $19,6(18,0-21,3)$ & $4,7 \quad(3,9-5,6)$ & $1,7(1,3-2,3)$ & $13,4(12,1-14,8)$ & $1,6(1,2-2,2)$ & $0,7(0,4-1,1)$ \\
\hline 40 a 59 anos & $38,3(36,0-40,6)$ & $25,4(23,4-27,5)$ & $8,8(7,6-10,3)$ & $9,6(8,4-10,8)$ & $3,9(3,20-4,8)$ & $2,2(1,8-2,8)$ \\
\hline 60 anos ou mais & $58,6(55,3-61,9)$ & $41,8(38,5-45,2)$ & $16,9(14,4-19,7)$ & $9,5(7,4-12,0)$ & $11,2(8,8-14,1)$ & $6,7(5,3-8,4)$ \\
\hline \multicolumn{7}{|l|}{ Escolaridade } \\
\hline Médio incompleto & $39,3(34,4-44,5)$ & $28,6(24,3-33,4)$ & $9,7(7,4-12,6)$ & $7,9(5,5-11,4)$ & $5,4(3,8-7,6)$ & $3,0(2,1-4,3)$ \\
\hline Médio completo & $34,0(32,3-35,7)$ & $18,5(17,2-20,0)$ & $7,2(6,3-8,3)$ & $12,1(11,0-13,3)$ & $4,4(3,7-5,4)$ & $2,3(1,8-2,9)$ \\
\hline Superior completo & $29,8(29,2-30,5)$ & $16,2(15,7-16,7)$ & $5,4(5,1-5,7)$ & $10,0(9,6-10,4)$ & $3,1(2,9-3,4)$ & $2,7(2,5-2,9)$ \\
\hline
\end{tabular}




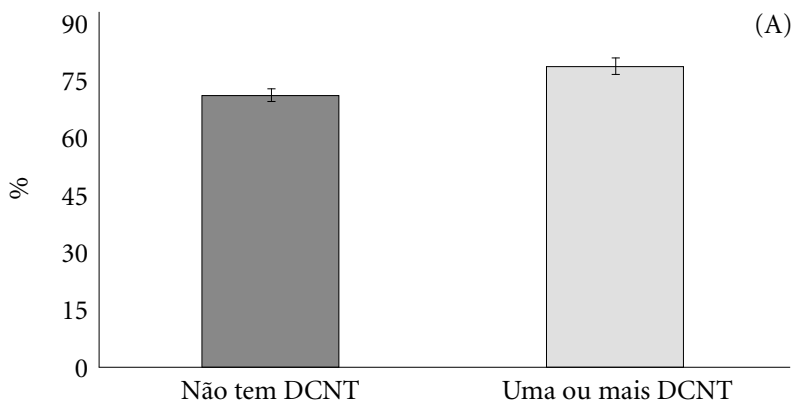

(A)

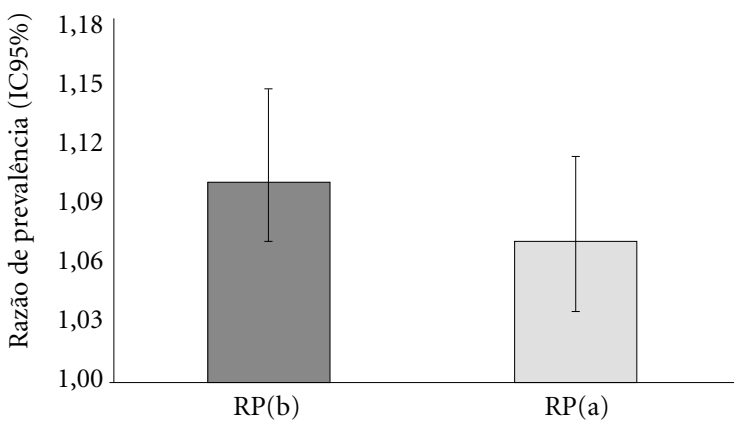

(B)

Figura 1. Prevalência (A) e razão de prevalência (B) da adesão ao distanciamento social intenso, segundo presença de DCNT. Brasil. ConVid - Pesquisa de Comportamentos, 2020.

Nota: DCNT = Doença Crônica Não Transmissível; RP (b) = Razão de Prevalência bruta; RP (a) = Razão de prevalência ajustada por sexo, faixa etária e escolaridade; IC95\% = Intervalo de confiança de 95\%.

A Tabela 3 apresenta as prevalências e as medidas de associação entre ter uma ou mais DCNT e a procura e dificuldade de utilização dos serviços de saúde durante a pandemia. Verificou-se que ter referido diagnóstico de DCNT esteve associado a maior procura por atendimento de saúde com um médico, dentista ou outro profissional de saúde (RPa: 1,24; IC95\%:1,11$1,38)$, a maior dificuldade no acesso aos serviços de saúde, como marcar consulta ( $\mathrm{RPa}$ : 1,52; IC95\%:1,35-1,71), conseguir atendimento de saúde (RPa: 1,50; IC95\%:1,22-1,84); conseguir medicamentos (RPa: 2,17; IC95\%: 1,77-2,67), realizar exames solicitados (RPa: 1,78; IC95\%: $1,50-2,10)$ e intervenções programadas (RPa: 1,65; IC95\%:1,16-2,34) (Tabela 3).

\section{Discussão}

O estudo identificou que cerca de $34 \%$ dos participantes referiu uma ou mais DCNT, entre hiper- tensão, diabetes, câncer, doença cardíaca ou doença respiratória. Em geral, a prevalência dessas doenças aumentou com a idade e foi mais elevada em populações com baixa escolaridade. Os indivíduos com DCNT referiram maior adesão às medidas de distanciamento social intenso, procuraram com maior frequência o atendimento de saúde, mas tiveram mais dificuldades no acesso aos serviços de saúde, como conseguir atendimento, marcar consulta, conseguir medicamentos, realizar exames e intervenções programadas.

A prevalência de diagnóstico prévio autorreferido de uma ou mais DCNT encontrada no presente estudo (34\%) foi inferior à revelada em publicação que analisou dados da Pesquisa Nacional de Saúde (PNS) de 2013 (45\%) ${ }^{14}$. Essa divergência pode ser explicada pelo fato deste estudo avaliar apenas cinco DCNT, ao passo que a PNS investigou um número maior. Ainda de acordo com a PNS 2013, em adultos, as prevalências de hipertensão arterial, diabetes, doença do coração e câncer foram, respectivamente, 21,4\%, 
Tabela 3. Prevalência e Razão de Prevalência bruta e ajustada da procura e dificuldade de utilização de serviços de saúde, segundo presença de DCNT. Brasil, ConVid - Pesquisa de Comportamentos, 2020.

\begin{tabular}{lcccc}
\hline \multicolumn{1}{c}{ Variáveis } & $\begin{array}{c}\text { Não ter DCNT } \\
\%(\mathbf{I C 9 5 \% )}\end{array}$ & $\begin{array}{c}\text { Ter uma ou } \\
\text { mais DCNT \% } \\
\text { (IC95\%) }\end{array}$ & $\begin{array}{c}\mathbf{R P ~ B r u t a}^{*} \\
(\mathbf{I C 9 5 \% )}\end{array}$ & $\begin{array}{c}\mathbf{R P ~ a j u s t a d a}^{\dagger} \\
(\mathbf{I C 9 5 \% )}\end{array}$ \\
\hline $\begin{array}{l}\text { Procurou atendimento de saúde } \\
\text { com um médico, dentista ou } \\
\text { outro profissional de saúde }\end{array}$ & $20,4(19,0-21,8)$ & $24,2(22,2-26,2)$ & $1,18(1,06-1,31)$ & $1,24(1,11-1,39)$ \\
$\begin{array}{l}\text { Dificuldade para marcar consulta } \\
\text { Dificuldade para conseguir } \\
\text { atendimento de saúde }\end{array}$ & $16,7(15,4-18,1)$ & $23,6(21,7-25,6)$ & $1,41(1,25-1,59)$ & $1,52(1,35-1,71)$ \\
$\begin{array}{l}\text { Dificuldade para conseguir } \\
\text { medicamentos }\end{array}$ & $8,2(7,2-9,4)$ & $11,2(9,6-12,9)$ & $1,36(1,11-1,65)$ & $1,50(1,22-1,84)$ \\
$\begin{array}{l}\text { Dificuldade para realizar exames } \\
\text { solicitados }\end{array}$ & $9,8(5,0-6,7)$ & $11,3(9,7-13,1)$ & $1,94(1,57-2,39)$ & $2,17(1,77-2,67)$ \\
$\begin{array}{l}\text { Dificuldade para realizar } \\
\text { intervenções programadas }\end{array}$ & $2,4(1,9-3,0)$ & $3,6(2,8-4,6)$ & $1,49(1,08-2,05)$ & $1,65(1,16-2,34)$ \\
$\begin{array}{l}\text { Dificuldade para conseguir vaga } \\
\text { para internação }\end{array}$ & $0,7(0,3-1,5)$ & $0,8(0,4-1,6)$ & $1,20(0,42-3,47)$ & $1,70(0,60-4,79)$ \\
\hline
\end{tabular}

Nota: ${ }^{\star}$ Razão de Prevalência bruta; ${ }^{\dagger}$ Razão de Prevalência ajustada por sexo, faixa etária e escolaridade; DCNT: Doença Crônica Não Transmissível; IC95\%: Intervalo de confiança de 95\%.

Fonte: ConVid - Pesquisa de Comportamentos, 2020.

$6,2 \%, 4,8 \%$ e $1,8 \%{ }^{14}$, próximas às encontradas no estudo atual.

Da mesma forma que no presente estudo, outra publicação que analisou dados da PNS 2013 identificou que a prevalência de DCNT aumentou com a idade, acometendo com maior frequência indivíduos com idade acima de 60 anos, exceto para asma, e de baixa escolaridade, exceto para câncer ${ }^{15}$. Para além das mudanças no estilo de vida das sociedades contemporâneas, fatores intrínsecos ao indivíduo e condições socioeconômicas, as doenças crônicas também guardam relação com o envelhecimento populacional ${ }^{16}$, por sua vez um fenômeno mundial ocasionado pelo declínio nas taxas de fertilidade e aumento da longevidade, ao qual se observa maior celeridade de ocorrência nos países menos desenvolvidos, embora os países mais desenvolvidos ainda detenham maior contingente de população idosa ${ }^{17-18}$. Estima-se que as DCNT respondam por $87 \%$ da carga de doenças entre a população com 60 anos e mais em países de baixa, média e alta renda ${ }^{17}$.

Os determinantes sociais, como escolaridade, renda e ambiente, também contribuem para aumento e gravidade das DCNT, dos seus fatores de risco e da morbimortalidade ${ }^{19}$. Indivíduos ou grupos de baixa renda e com menos escolaridade são mais prováveis de estarem mais expostos a fatores de risco para as DCNT, haja vista terem menos acesso ou acesso inadequado aos serviços de saúde, a uma alimentação balanceada e a ambientes saudáveis ${ }^{20-21}$, o que reduz as oportunidades de promoção da saúde e prevenção de doenças ${ }^{22}$.

Cabe destacar que a pandemia de COVID-19 está ocorrendo em um cenário de desigualdades sociais e econômicas e pode piorar a morbimortalidade por DCNT, agravando as iniquidades em saúde ${ }^{23}$. A prevalência e a gravidade da pandemia de COVID-19 são ampliadas devido à distribuição desigual das DCNT e seus fatores de risco, afetando mais as populações vulneráveis, o que denota estreita relação com os determinantes sociais da saúde ci-25. $^{23}$.

A adesão ao distanciamento social intenso foi mais elevada entre os indivíduos com DCNT. Estes achados podem ser explicados pelo medo de adoecimento e pelo sentimento de maior vulnerabilidade desta população, que opta por seguir as orientações de distanciamento social de forma mais rigorosa, buscando se proteger ${ }^{7}$. As medidas de distanciamento social contribuem para redução do risco de transmissão da COVID-19 para toda a população, em especial, para aqueles com DCNT, que têm maior risco de agravamento dos casos e mortalidade ${ }^{2,26}$. 
O presente estudo apontou que os indivíduos com DCNT referiram maior procura e dificuldades na utilização de serviços de saúde durante a pandemia. Os determinantes da utilização dos serviços de saúde estão relacionados, entre outros fatores, com a necessidade de saúde da população, delineada pela morbidade, gravidade ou urgência da doença ${ }^{27}$. As DCNT, dada sua elevada carga e cronicidade, estão entre as doenças que demandam mais ações, procedimentos e serviços de saúde ${ }^{28}$. Nesse sentido, um estudo que utilizou dados da PNS em 2013 evidenciou que a presença de ao menos uma doença crônica estava associada ao maior uso de serviços de saúde $(25,6 \%)$, em comparação à ausência de DCNT $(10,8 \%)^{29}$. Além disso, a presença de DCNT associou-se ao aumento de internação ${ }^{29}$. A COVID-19 evidenciou a alta carga que tais doenças representam para os recursos e serviços de saúde, exigindo dos governos a implementação mais rígida de ações para controle do tabaco, álcool e açúcar, bem como investimentos para melhoria da atividade física e dietas saudáveis ${ }^{30}$.

As dificuldades na utilização dos serviços mencionadas pelos participantes deste estudo podem ser explicadas pela menor oferta de serviços pelos setores de saúde público e privado durante a pandemia. Esforços para lidar com a COVID-19 também interromperam os cuidados regulares habitualmente exigidos para indivíduos com DCNT ${ }^{30}$. Pesquisa feita pela OMS constatou que $75 \%$ dos países respondentes relataram interrupções nos serviços de prevenção e tratamento de $\mathrm{DCNT}^{31}$.

A interrupção ou redução da utilização dos serviços de saúde de rotina e de suprimentos médicos pioram o acompanhamento e manejo das DCNT e aumentam a morbidade, incapacidade e mortalidade evitável ${ }^{2}$. Estudo realizado pela OPAS em maio de 2020, entre os países das Américas, também identificou que, embora os serviços ambulatoriais de DCNT estivessem mantidos, ocorreu acesso limitado em vários países $(64 \%)^{8}$. A interrupção dos serviços, parcial ou totalmente, afetou todos os tipos de atendimento para pessoas com DCNT, como odontológico e serviços de reabilitação ${ }^{8}$. Os principais motivos citados nas respostas dos estados membros para a interrupção dos serviços de DCNT incluíram: o cancelamento dos serviços de atendimento eletivo $(58 \%)$, a realocação da equipe clínica para a resposta ao COVID-19 (50\%) e o não comparecimento dos indivíduos aos atendimentos de saúde $(50 \%)^{8}$. A redução do transporte público ocorrida durante a pandemia também pode di- ficultar a utilização de serviços e interromper a continuidade dos cuidados para portadores de $\mathrm{DCNT}^{2}$. Sobre o não comparecimento de pacientes/clientes/usuários aos atendimentos, publicação que investigou o uso de serviços de saúde nos primeiros dois meses de pandemia por população segurada nos Estados Unidos identificou redução significativa do uso de serviços eletivos e preventivos e aumento da telemedicina ${ }^{32}$. Esse não comparecimento aos serviços de saúde pode ter como uma possível explicação o receio de exposição a maior risco de contrair COVID-1933.

Entre as limitações do estudo encontra-se a coleta de dados pela internet, estratégia que pode não atingir todos os estratos populacionais, pois considera-se que nem todos têm acesso a este meio de comunicação. Esse fato pode levar a sub ou superestimação da proporção dos indicadores investigados. Essa limitação, contudo, foi minimizada devido ao grande número amostral e à calibração da amostra com os dados da PNAD de 2019. Cabe destacar que a coleta de dados via internet e o processo de amostragem em cadeia é o único método eticamente plausível, no contexto da pandemia, para avaliação das questões de interesse. É, também, um método de pesquisa que apresenta baixo custo relacionado a sua operacionalização. As pesquisas online surgem como um método promissor para avaliar e rastrear conhecimentos, comportamentos, estilos de vida e percepções durante surtos de doenças infecciosas em rápida evolução. Outra questão diz respeito à realização da coleta de dados no início da pandemia (precisamente, no terceiro mês), o que reflete, portanto, o retrato de um momento específico, sujeito a mudanças conjunturais ao longo da evolução temporal da epidemia de COVID-19 no Brasil. Adicionalmente, em virtude de as informações coletadas terem sido autorreferidas, pode haver viés de informação concernente ao diagnóstico prévio de DCNT. Ressalta-se, ainda, a utilização de apenas variáveis socidemográficas para ajuste dos modelos, o que pode resultar em potencial confusão residual.

\section{Conclusão}

A pandemia resultou em importante adesão dos indivíduos com DCNT ao distanciamento social, entretanto, observa-se, para esse segmento populacional, maior procura e dificuldade na utilização dos serviços de saúde. Assim, reforça-se a importância da discussão acerca das políticas de enfrentamento das doenças crônicas e das ações 
de cuidado face à conjuntura da pandemia de COVID-19. As medidas de prevenção de DCNT não devem ser interrompidas, os serviços de saúde, em especial a Atenção Primária à Saúde, precisam adaptar-se à nova realidade para apoiar e gerenciar o aumento dos riscos das pessoas e dar continuidade aos cuidados a portadores de
DCNT. A equipe de saúde deve estar envolvida no planejamento de estratégias de resposta à COVID-19 para garantir que as necessidades dos pacientes sejam atendidas. Aconselhamentos específicos devem ser disponibilizados nacional e localmente para indivíduos com DCNT, suas famílias e cuidadores.

\section{Colaboradores}

Todos os autores trabalharam na concepção e no delineamento do estudo, na análise dos dados, na redação e revisão crítica do artigo e na aprovação da versão a ser publicada.

\section{Financiamento}

Fundo Nacional de Saúde, Ministério da Saúde (TED 66/2018). 


\section{Referências}

1. World Health Organization (WHO). Noncommunicable diseases country profiles 2018. Geneva: WHO; 2018 [cited 2021 Jan 19]:223 p. Available from: https:// www.who.int/nmh/publications/ncd-profiles-2018/ en/

2. Kluge HHP, Wickramasinghe K, Rippin HL, Mendes R, Peters DH, Kontsevaya A, Breda J. Prevention and control of non-communicable diseases in the COVID-19 response. Lancet 2020; 395(10238):16781680.

3. Istituto Superiore di Sanità. Characteristics of SARSCoV-2 patients dying in Italy. Report based on available data on September 7th, 2020. Roma: EpiCentro; 2020. [cited 2021 Jan 23]:1-10. Available from: https:// www.epicentro.iss.it/en/coronavirus/bollettino/Report-COVID-2019_7_september_2020.pdf

4. World Health Organization (WHO). Overview of public health and social measures in the context of COVID-19. Geneva: WHO; 2020 [cited 2021Jan 23]. Available from: https://www.who.int/publications/i/ item/overview-of-public-health-and-social-measures -in-the-context-of-covid-19

5. Malta DC, Szwarcwald CL, Barros MBA, Gomes CS, Machado IE, Souza Júnior PRB, Romero DL, Lima MG, Damacena GM, Pina MF, Freitas MIF, Werneck AL, Silva DRP, Azevedo LO, Gracie R. A pandemia da COVID-19 e as mudanças no estilo de vida dos brasileiros adultos: um estudo transversal, 2020. Epidemio Serv Saude 2020; 29(4): e2020407.

6. World Health Organization (WHO). Noncommunicable diseases in emergencies. Geneva: WHO; 2020 [cited 2021 Jan 23]. Available from: https://www.who.int/ ncds/publications/ncds-in-emergencies/en/

7. Barone MTU, Villarroel D, Luca PV, Harnik SB, Lima BLS, Wieselberg RJP, Giampaoli V. COVID-19 impact on people with diabetes in South and Central America (SACA region). Diabetes Res Clin Pract 2020; 166:108301.

8. Organização Pan-Americana da Saúde (OPAS). Informe de la evaluación rápida de la prestación de servicios para enfermedades no transmisibles durante la pandemia de COVID-19 en las Américas; 2020 [acessado 2021 Jan 23]:1-8. Disponível em: https://iris.paho.org/ bitstream/handle/10665.2/52283/OPSNMHNVCOVID-19200024_spa.pdf?sequence $=5$ \&isAllowed $=\mathrm{y}$

9. Fundação Oswaldo Cruz (Fiocruz). Convid Pesquisa de Comportamentos [Internet]. 2020 [acessado 2021 jan 23]. Disponível em: https://convid.fiocruz.br/index.php?pag=principal

10. Costa BRL. Bola de Neve Virtual: O Uso das redes sociais virtuais no processo de coleta de dados de uma pesquisa científica. Rev Interdiscip Gestão Soc 2018;7(1):15-37.

11. Szwarcwald CL, Damacena GN. Amostras complexas em inquéritos: planejamento e implicações na análise estatística de dados. Rev Bras Epidemio 2008; 11(Supl. 1):38-45

12. Instituto Brasileiro de Geografia e Estatística (IBGE). Pesquisa Nacional por Amostra de Domicílios (PNAD) 2019. Rio de Janeiro: IBGE; 2019 [acessado 2021 jan 23]. Disponível em: https://biblioteca.ibge.gov.br/visualizacao/livros/liv101651_notas_tecnicas.pdf
13. Bernal RTI, Iser BPM, Malta DC, Claro RM. Surveillance System for Risk and Protective Factors for Chronic Diseases by Telephone Survey (Vigitel): changes in weighting methodology. Epidemiol Serv Saude 2017; 26(4):701-712.

14. Malta DC, Stopa SR, Szwarcwald CL, Gomes NL, Silva Júnior JB, Reis AAC. A vigilância e o monitoramento das principais doenças crônicas não transmissíveis no Brasil - Pesquisa Nacional de Saúde, 2013. Rev Bras Epidemiol 2015;18 (Supl. 2):3-16.

15. Theme FMM, Souza JPRB, Damacena GN, Szwarcwald CL. Prevalência de doenças crônicas não transmissíveis e associação com autoavaliação de saúde: Pesquisa Nacional de Saúde, 2013. Rev Bras Epidemiol 2015; 18(Supl. 2): 83-96.

16. Veras RP. Estratégias para o enfrentamento das doenças crônicas: um modelo em que todos ganham. Rev Bras Geriatr Gerontol 2011; 14(4):779-786.

17. World Health Organization (WHO). Global Health and Aging. Geneva: WHO; 2011 [cited 2021 Jan 23]:132. Available from: https://www.who.int/ageing/publications/global_health.pdf?ua=1

18. Carvalho JAM, Garcia RA. O envelhecimento da população brasileira: um enfoque demográfico. Cad Saude Publica 2003; 19(3):725-733.

19. Marmot M, Bell R. Social determinants and noncommunicable diseases: time for integrated action BMJ 2019; 364: 1251.

20. Keetile M, Navaneetham K, Letamo G, Rakgoasi SD. Socioeconomic inequalities in non-communicable disease risk factors in Botswana: a cross-sectional study. BMC Public Health 2019; 19(1):1060.

21. World Health Organization (WHO). Preventing Cronic Diseases - a vital investment. Geneva: WHO; 2005 [cited 2021 Jan 23]. Available from: https://www.who. int/chp/chronic_disease_report/contents/en/

22. Malta DC, Moura EC, Morais Neto OL. Desigualdades de sexo e escolaridade em fatores de risco e proteção para doenças crônicas em adultos Brasileiros, por meio de inquéritos telefônicos. Rev Bras Epidemio 2011; 14(Supl.1):125-135.

23. Malta DC, Bernal RTI, Lima MG, Araújo SSC, Silva MMA, Freitas MIF, Barros MBA. Noncommunicable diseases and the use of health services: analysis of the National Health Survey in Brazil. Rev Saude Publica 2017; 51(Supl. 1):4s.

24. Barros MBA, Zanchetta LM, Moura EC, Malta DC. Auto-avaliação da saúde e fatores associados, Brasil, 2006. Rev Saude Publica 2009; 43(Supl. 2):27-37.

25. Bambra C, Riordan R, Ford J, Matthews F. The COVID-19 pandemic and health inequalities. J Epidemiol Community Health 2020; 74:964-968.

26. Nussbaumer-Streit B, Mayr V, Dobrescu AI, Chapman A, Persad E, Klerings I, Wagner G, Siebert U, Christof C, Zachariah C, Gartlehner G. Quarantine alone or in combination with other public health measures to control COVID-19: a rapid review. Cochrane Database Syst Rev 2020; 4(4):CD013574.

27. Travassos C, Martins M. Uma revisão sobre os conceitos de acesso e utilização de serviços de saúde. Cad Saude Publica 2004; 20(Supl. 2):S190-S198. 
28. Brasil. Ministério da Saúde (MS). A vigilância, o controle e a prevenção das doenças crônicas não-transmissiveis: DCNT no contexto do Sistema Único de Saúde brasileiro. Brasília: Organização Pan-Americana da Saúde [Internet]. 2005 [acessado 2021 jan 23]:1-80. Disponível em: https://bvsms.saude.gov.br/bvs/publicacoes/DCNT.pdf

29. Malta DC, Bernal RTI, Lima MG, Araújo Silvânia SC, Silva MMA, Freitas MIF, Barros MB. Doenças crônicas não transmissíveis e a utilização de serviços de saúde: análise da Pesquisa Nacional de Saúde no Brasil. Rev Saude Publica 2017; 51(Supl. 1):4s.

30. The Lancet. COVID-19: a new lens for non-communicable diseases. Lancet 2020; 396 (10252):649.

31. World Health Organization (WHO). NCD Department. Rapid assessment of service delivery for noncommunicable diseases (NCDs) during the COVID-19 pandemic: Final Results. Geneva: WHO; 2020 [cited 2021 Jan 23]. Available from: https://www.who.int/ publications $/ \mathrm{m} /$ item/rapid-assessment-of-servicedelivery-for-ncds-during-the-covid-19-pandemic

32. Whaley CM, Pera MF, Cantor J, Chang J, Velasco J, Hagg HK, Sood N, Bravata DM. Changes in Health Services Use Among Commercially Insured US Populations During the COVID-19 Pandemic. JAMA Netw Open 2020;3(11):e2024984.

33. Carlos RC, Lowry KP, Sadigh G. The Coronavirus Disease 2019 (COVID-19) Pandemic: A Patient-Centered Model of Systemic Shock and Cancer Care Adherence. J Am Coll Radiol 2020; 17(7):927-930.

Artigo apresentado em 06/10/2020

Aprovado em 22/03/2021

Versão final apresentada em 24/03/2021

Editores-chefes: Romeu Gomes, Antônio Augusto Moura da Silva 